gene expression in mouse myocardium. J Thorac Cardiovasc Surg. 2005;130:1326-32.

5. Konstantinov IE, Li J, Cheung MM, Shimizu M, Stokoe J, Kharbanda RK, et al. Remote ischemic preconditioning of the recipient reduces myocardial ischemia-reperfusion injury of the denervated donor heart via a Katp channel-dependent mechanism. Transplantation. 2005;79:1691-5.

doi:10.1016/j.jtcvs.2005.07.043

\section{From mesothelioma to}

cardiovascular protection via the phosphoinositide-3 kinase pathway: A new vista in cardiothoracic

\section{surgery}

\section{To the Editor:}

We read with interest the article by Rascoe and colleagues ${ }^{1}$ on the role of tyrosine kinase receptors and the phosphoinositide-3 kinase (PI3K) pathway in mesothelioma. The authors demonstrated differential involvement of 3 growth factor (GF) receptors-epidermal growth factor (EGF), insulin-like growth factor (IGF), and platelet-derived growth factor (PDGF) receptors-in PI3K-mediated survival of the malignant mesothelioma cells.
Survival of many cells under stress, particularly of myocytes, is dependent on PI3K pathway activation. ${ }^{2}$ It is not often appreciated, however, that the PI3K pathway can be activated not only via tyrosine kinase receptors by GFs, but also via toll-like receptor by heat shock protein, bacterial lipopolysaccharide, and by tumor necrosis factor (Figure 1). Although the precise molecular mechanisms by which the GF receptors interact with $\mathrm{PI} 3 \mathrm{~K}$ pathway are unknown, they appear to have different properties. For example, PDGF appears to play a crucial role in the pathogenesis of atherosclerosis. ${ }^{3}$ We agree with Rascoe and colleagues that PDGF receptor inhibition does not negatively affect cellular survival. In fact, PDGF inhibition results in significant reduction in oxidative stress ${ }^{3}$ and is observed after ischemic preconditioning. ${ }^{4}$ Indeed, we have previously demonstrated that intravenous infusion of IGF improves oxygen transport by reducing oxygen consumption as well as increasing cardiac output and oxygen delivery after ischemia-reperfusion (IR) injury. ${ }^{5}$ Further- more, cardiovascular protection from IR injury is abrogated when PI3K pathway is inhibited by LY294002. ${ }^{2}$ Thus, systemic inhibition of IGF or PI3K may result in detrimental clinical side effects.

While local inhibition of PI3K signaling either directly or via EGF receptor inhibition may appear a promising treatment for mesothelioma, it is possible that sustained systemic inhibition of PI3K, either directly by LY294002 or indirectly via tyrosine kinase receptor inhibition, could lead to rapid progression of vascular disease and increased myocardial vulnerability to IR syndromes in susceptible patients. Whatever the clinical implications of the study by Rascoe and colleagues might be, any therapeutic intervention must be applied cautiously and balanced carefully against potential risks.

In summary, we congratulate Rascoe and colleagues on their stimulating and thoughtprovoking study. It is clear that contemporary academic cardiothoracic surgery must be performed within the realms of an understanding of the burgeoning field of molecular

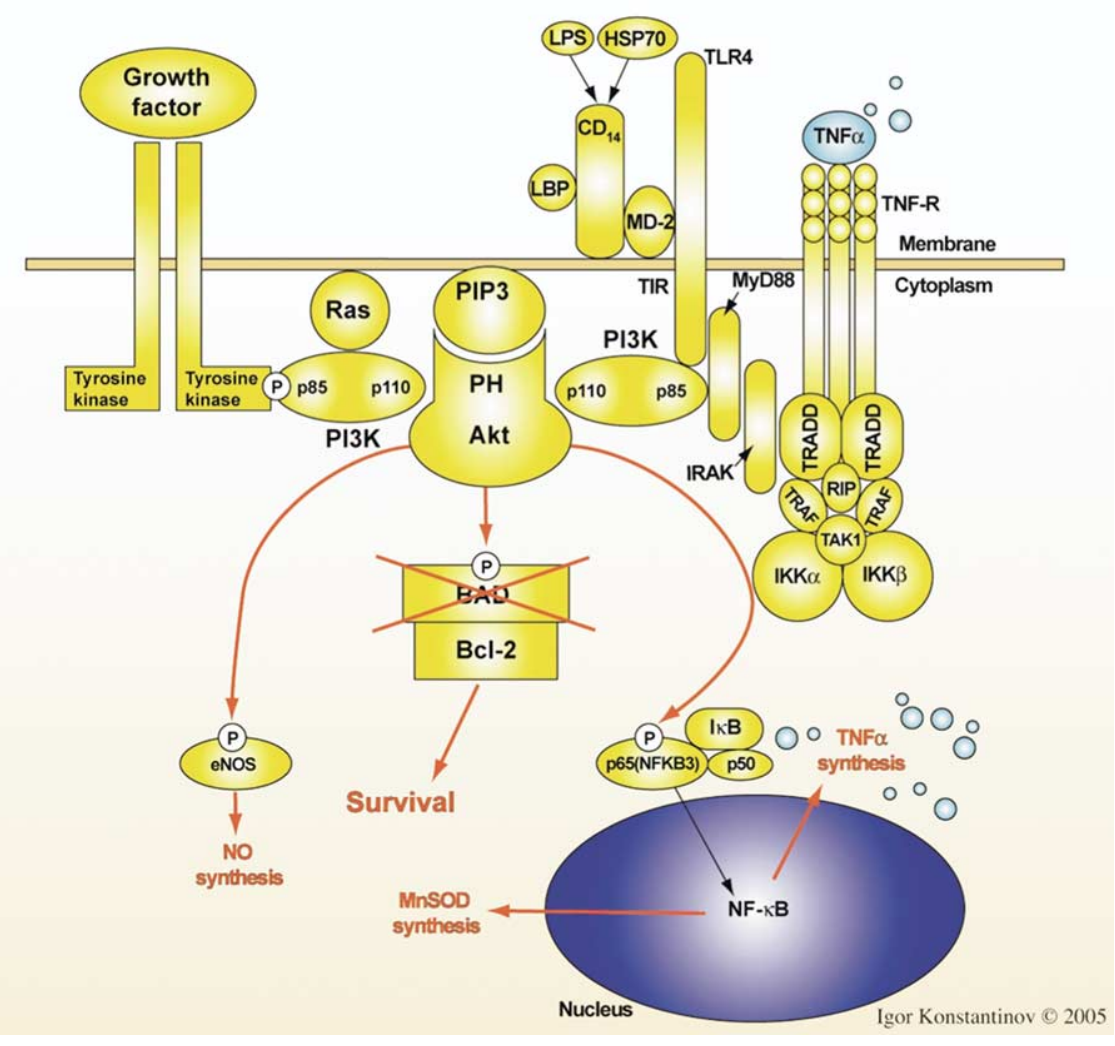

Figure 1. Schematic of phosphoinositide-3 kinase (PI3K) signaling pathway. Activation of growth factor receptor results in phosphorylation $(P)$ on tyrosine residues. PI3K is activated by binding to tyrosine residues of growth factor receptor with its regulatory subunit (p85). The lipid product of PI3K, phosphadylinositol-3,4,5-triphosphate (PIP3), recruits serine-treonine kinase (Akt) via pleckstrin homology (PH) domain to the membrane, where it is activated. Akt phosphorylation of the apoptosis inducing protein $B A D$ prevents BAD from binding to Bcl-2, thus releasing it for survival response. Direct binding to Ras protein further stimulates PI3K activity. Stimulation of toll-like receptor (TLR) 4 by bacterial lipopolysaccharide (LPS) or heat shock protein (HSP) results in PI3K activation via the myeloid differentiation factor (MyD) 88. Tumor necrosis factor (TNF) produces PI3K/ Akt-mediated phosphorylation of p65 subunit of nuclear factor kappaB (NF-кB) via the alpha subunit of lkappaB kinase (IKK). Translocation of NF-kB to the nucleus results in gene expression and systhesis of cytokines, manganese superoxide dismutase (MnSOD), and endothelial nitric oxide synthase (eNOS) that are protective during acute ischemia. 
biology and its relevance to clinical therapy. This is a laudable step along that path. Igor E. Konstantinov, $M D, P h D$

Jia $\mathrm{Li}, \mathrm{MD}, \mathrm{PhD}$

Andrew N. Redington, MD

Department of Cardiovascular Surgery and Cardiology

Hospital for Sick Children Toronto, Ontario, Canada

\section{References}

1. Rascoe PA, Cao X, Daniel JD, Miller SD, Smythe WR. Receptor tyrosine kinase and phosphoinositide-3 kinase signaling in malignant mesothelioma. J Thorac Cardiovasc Surg. 2005;130:393-400.

2. Hausenloy DJ, Tsang A, Mocanu MM, Yellon DM. Ischemic preconditioning protects by activating prosurvival kinases at reperfusion. Am J Physiol Heart Circ Physiol. 2005; 288:H971-6.

3. Rosenkranz S, Knirel D, Dietrich H, Flesch M, Erdmann E, Bohn M. Inhibition of the PDGF receptor by red wine flavonoinds provides a molecular explanation for the French paradox. FASEB J. 2002;16:1958-60.

4. Konstantinov IE, Arab S, Li J, Coles JG, Boscarino C, Mori A, et al. The remote ischemic preconditioning stimulus modifies gene expression in mouse myocardium. $J$ Thorac Cardiovasc Surg. 2005;130:1326-32.
5. Li J, Stenbog E, Bush A, Grofte T, Redington AN, Penny DJ. Insulin-like growth factor 1 improves the relationship between systemic oxygen consumption and delivery in piglets after cardiopulmonary bypass. J Thorac Cardiovasc Surg. 2004;127:1436-41. doi:10.1016/j.jtcvs.2005.09.058

\section{Reply to the Editor:}

We greatly appreciate the kind remarks and insightful comments by Konstantinov, $\mathrm{Li}$, and Reddington concerning our recent manuscript, "Receptor Tyrosine Kinase and Phosphoinositide-3 Kinase Signaling in Malignant Mesothelioma."

Although constitutive activation of the PI3K/Akt pathway has been demonstrated in a number of solid malignancies, its importance is certainly not limited to neoplastic cells. In fact, Akt signaling enhances vital cellular processes such as glycogen synthesis and nitric oxide production. LY294002 is a lead compound in the development of PI3K inhibitors and is not available for human trials. However, we believe that further drug development could possibly produce a compound with acceptable toxicity and therapeutic potential in oncology. We agree that any new interventions must be applied with caution in the context of a controlled trial.

Similarly, inhibitors of the insulin-like growth factor receptor are still in the developmental phase. While such compounds have potential as antineoplastic agents, there is also the potential for toxic effects such as the development of endocrinopathies. However, inhibitors of receptor tyrosine kinases such as epidermal growth factor receptor (Iressa, Tarceva) and platelet-derived growth factor receptor (Gleevec) are currently used in the treatment of solid and hematologic malignant tumors with acceptable side effect profiles.

We again thank Konstantinov, Li, and Reddington for their interest in our work. It is clear that an understanding of molecular mechanisms will be requisite for contemporary clinicians, particularly those treating cardiovascular and oncologic disease.

Philip A. Rascoe, $M D$

Xiaobo Cao, MD

Jonathan C. Daniel, MD

Steven D. Miller, MD

W. Roy Smythe, MD

M.D. Anderson Cancer Center

Houston, TX 77030

doi:10.1016/j.jtcvs.2005.11.019 